\title{
Yeast prions assembly and propagation Contributions of the prion and non-prion moieties and the nature of assemblies
}

\author{
Mehdi Kabani and Ronald Melki \\ Laboratoire d'Enzymologie et Biochimie Structurales; Centre National de la Recherche Scientifique; Gif-sur-Yvette, France
}

Key words: prion, Sup35p, Ure2p, Rnq1p, [PSI+], [URE3], [PIN+], amyloid fibrils

Yeast prions are self-perpetuating protein aggregates that are at the origin of heritable and transmissible non-Mendelian phenotypic traits. Among these, $\left[P S I^{+}\right],[U R E 3]$ and $\left[P I N^{+}\right]$are the most well documented prions and arise from the assembly of Sup35p, Ure2p and Rnq1p, respectively, into insoluble fibrillar assemblies. Fibril assembly depends on the presence of $\mathrm{N}$ - or C-terminal prion domains (PrDs) which are not homologous in sequence but share unusual amino-acid compositions, such as enrichment in polar residues (glutamines and asparagines) or the presence of oligopeptide repeats. Purified PrDs form amyloid fibrils that can convert prion-free cells to the prion state upon transformation. Nonetheless, isolated PrDs and fulllength prion proteins have different aggregation, structural and infectious properties. In addition, mutations in the "nonprion" domains (non-PrDs) of Sup35p, Ure2p and Rnq1p were shown to affect their prion properties in vitro and in vivo. Despite these evidences, the implication of the functional non-PrDs in fibril assembly and prion propagation has been mostly overlooked. In this review, we discuss the contribution of non-PrDs to prion assemblies, and the structure-function relationship in prion infectivity in the light of recent findings on Sup35p and Ure2p assembly into infectious fibrils from our laboratory and others.

\section{Introduction}

Yeast prions are self-perpetuating fibrillar aggregates that manifest as dominant traits which are cytoplasmically inherited in a non-Mendelian manner. $\left[P S I^{+}\right],[U R E 3]$ and $\left[P I N^{+}\right]$, which are the prion forms of Sup35p, Ure2p and Rnq1p, respectively, are the best known and the most documented yeast prions. ${ }^{1,2}$ The biological significance of prions for fungi in their natural ecological niches has been recently reviewed in references 1-3.

Yeast prion proteins are not homologous. The striking enrichment in proteins involved in transcriptional and translational control led to the idea that prions, in yeast, may act as epigenetic modifiers of gene expression, enabling yeasts to rapidly

Correspondence to: Mehdi Kabani and Ronald Melki;

Email: Mehdi.Kabani@lebs.cnrs-gif.fr and Ronald.Melki@lebs.cnrs-gif.frt Submited: 08/12/11; Accepted: 09/13/11

DOI: $10.4161 /$ pri.5.4.18070 (and transiently) adapt to changes in their surrounding environment. ${ }^{1-3}$ While this hypothesis can be successfully tested in laboratory strains and conditions, the inability to identify wild-type yeast isolates that stably propagate one of the well-known prions raises doubts on whether this is actually a real mechanism for the control of gene expression in the natural environment. ${ }^{4}$

Under physiological conditions, prion proteins spontaneously assemble into fibrils in vitro (Fig. 1). When re-introduced into prion-free yeast cells, these fibrils are able to induce their conversion to the prion state with high efficiency. ${ }^{5-9}$ Fibril formation depends on the presence of a domain, often referred to as the prion domain $(\mathrm{PrD})$, essential for assembly and the conversion of the protein from the soluble, functional state to the aggregated prion state. In most cases, the $\operatorname{PrD}$ is dispensable for the normal function of the protein. ${ }^{10,11}$ Although diverse, the PrDs share common characteristics such as enrichment in glutamines and asparagines, and the presence of oligopeptide repeats (Fig. 2). These traits are reminiscent of similar motifs found in the mammalian prion PrP, responsible for Creutzfeld-Jakob disease, sheep scrapie and other transmissible spongiform encephalopathies, as well as in proteins responsible for neurodegenerative diseases such as Huntington disease or spinocerebellar ataxia, ${ }^{12,13}$ which have recently been shown to have prion-like properties that may play a significant role in neurodegenerative disease progression. ${ }^{14-22}$

The PrDs can be found at either the N- or C-termini of prion proteins (Fig. 2). Purified PrDs are able to assemble into amyloid-like fibrils (Fig. 1) that can convert prion-free cells to the prion state upon transformation. ${ }^{6,8,9,23-25} \mathrm{PrDs}$ can assemble into different structural variants that induce the formation of different prion strains, akin to mammalian prion strains, with varying phenotypic strength, mitotic stability and susceptibility to the action of molecular chaperones. ${ }^{6,8,9}$ Finally, non-prion proteins acquire a propensity to aggregate when fused to PrDs. ${ }^{26-28}$ Altogether, experimental evidence supports a model where $\mathrm{PrDs}$ are the sole drivers of prion formation and propagation.

However, this model is challenged by many studies demonstrating that isolated $\mathrm{PrDs}$ and full-length prion proteins have different aggregation, structural and infectious properties, and that mutations in the 'non-prion' domains (non-PrDs) of yeast prion proteins affect their prion properties. ${ }^{711,29,30}$ In this review, we discuss the contribution of non-PrDs to prion assemblies and the structure-function relationship in prion infectivity in 


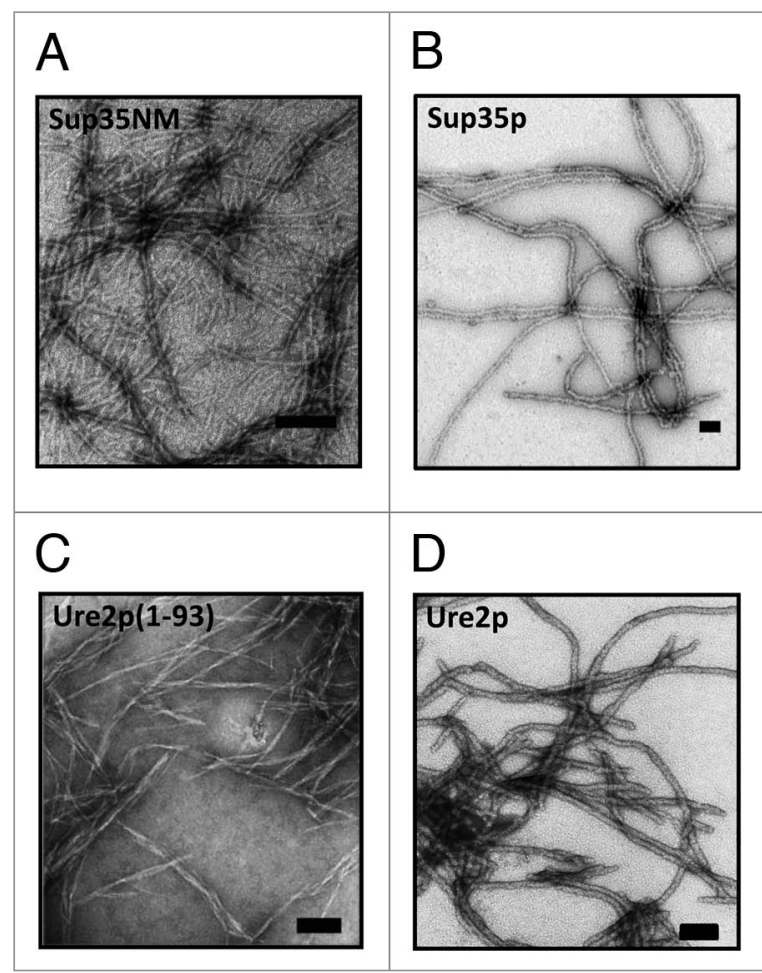

Figure 1. Electron micrographs of negatively stained (A) Sup35NM, (B) full-length Sup35p, (C) Ure2p (1-93) and (D) full-length Ure2p fibrils (scale bar: $0.1 \mu \mathrm{m}$ ).

the light of recent findings on Sup35p and Ure2p assembly into infectious fibrils from our laboratory and others.

\section{The $\left[P S{ }^{+}\right]$and [URE3] Prions}

The non-Mendelian $\left[\mathrm{PSI}^{+}\right]$and [URE3] traits were described more than three decades ago and were subsequently shown to be the prion forms of Sup35p and Ure2p, respectively. ${ }^{31-35}$ Sup35p (Fig. 2) is a component of the eukaryotic release factor, homologous to eRF3, which mediates stop codon recognition and translation termination in conjunction with Sup45p (eRF1). In $\left[\mathrm{PSI}^{+}\right]$cells a substantial proportion of soluble Sup35p is depleted within prion aggregates, resulting in an increased nonsense suppression phenotype due to defective translation termination. It is worth noting that de novo $\left[\mathrm{PSI}^{+}\right]$formation, but not propagation, requires the presence of $\left[\mathrm{PIN}^{+}\right] .^{36-38}$

Ure2p (Fig. 2) is a negative regulator of nitrogen catabolism. When good nitrogen sources such as ammonia are present in the growth medium, Ure2p represses the Gln3p transcriptional activator that controls the expression of genes encoding enzymes and transporters required for the assimilation of poor nitrogen sources. ${ }^{39-41}$ In [URE3] cells, Ure2p is sequestered within prion aggregates and can no longer interact with Gln $3 p$, which becomes constitutively activated. [URE3] cells are therefore able to metabolize poor nitrogen sources such as allantoate, and because of its structural analogy, ureidosuccinate, even in the presence of good nitrogen sources. ${ }^{34}$
Both $\left[\mathrm{PSI}^{+}\right]$and [URE3] are cured by treatment with millimolar concentrations of guanidine hydrochloride or by deleting HSP104, but only $\left[\mathrm{PSI}^{+}\right]$can be cured by the overexpression of HSP104. ${ }^{42,43}$

\section{Biological Functions of the Prion and "Non-Prion" Domains of Sup35p and Ure2p}

What is the contribution of prion domains to the normal cellular function of Sup35p and Ure2p? Several lines of evidence suggest that prion propagation and normal cellular functions are carried out by separate, well defined regions within Sup35p and Ure2p (Fig. 2). Cells in which the essential SUP35 gene has been replaced by a sup35 allele where the N-terminal (Sup35N; residues 1-123) or NM (Sup35NM; residues 1-253) domains have been deleted grow as well as the wild-type, suggesting that only the C-terminal domain is required for the function of Sup35p in translation termination. ${ }^{10}$ Similarly, an ure 2 allele where the $\mathrm{N}$-terminal domain (residues 1-89) has been deleted can perfectly restore nitrogen catabolism regulation in a $\Delta$ ure 2 strain. ${ }^{11}$ However, these strains are not able to convert to the prion state or maintain it. Conversely, the transient overexpression of Sup $35 \mathrm{~N}$ and Sup35NM or residues 1-89 from Ure2p efficiently induces $\left[\mathrm{PSI}^{+}\right]$and [URE3], respectively. ${ }^{11,44}$ Sup35N, Sup35NM and Ure $2 \mathrm{p} N$-terminal fragments encompassing residues 1-89 are able to assemble into amyloid-like fibrils in vitro (Fig. 1) that can efficiently induce the $\left[\mathrm{PRION}^{+}\right]$state upon transformation into [prion ${ }^{-}$cells. ${ }^{6,9,30}$

However, these boundaries have been blurred by other observations that suggest that functional and/or physical interaction between prion and 'non-prion' domains may contribute to both the prion and normal cellular functions of Sup35p and Ure2p. Indeed, the C-terminal domain of Sup35p is strongly conserved through evolution from yeast to mammals. ${ }^{45}$ The $\mathrm{N}$-terminal extension is present in most Sup35p/eRF3 orthologs suggesting that it plays an important, yet unclear, role in the function(s) of these proteins. ${ }^{45,46}$ Although highly divergent in length and sequence, the amino-acid composition of the $\mathrm{N}$-terminal domain of Sup35/eRF3 proteins from yeast to mammals is often highly unusual (e.g., mostly enriched in $\mathrm{Q} / \mathrm{N}$ in yeasts and $\mathrm{P} / \mathrm{G} / \mathrm{S}$ in higher eukaryotes)..$^{13,46,47}$ The only crystallographic structure of eRF3 available is that of Schizosaccharomyces pombe's Sup35p which suggests that the $\mathrm{N}$-terminal extension could bind and block the eRF1 binding site in the C-terminal domain (Fig. 2). ${ }^{48}$ Accordingly, both negative and positive effects of the $\mathrm{N}$-terminal domain of yeast, fungal and mammalian eRF3 proteins on translation termination, non-sense suppression and eRF1 binding were described. ${ }^{7,49-54}$

Similarly, the globular C-terminal domain of Ure2p (residues 90-354) is dimeric in solution, displays homology to glutathione S-tranferases (Fig. 2) and binds glutathione or related compounds with high affinity. ${ }^{39,55-57}$ This globular domain retains its native structure in the fibrillar form of the protein..$^{58-60}$ While the C-terminal domain alone can support the nitrogen regulation function of Ure $2 p$ (and complement a $\Delta u r e 2$ allele), the 
presence of the N-terminal domain appears to be required for tighter nitrogen catabolite repression under normal Ure2p expression levels. ${ }^{39,61,62}$ Ure $2 p$ displays glutathione peroxidase and glutaredoxin activities, in both the soluble and fibrillar state, and therefore contributes to cell protection against heavy metal ions and oxidant toxicity. ${ }^{63-65}$ However, truncations within the $\mathrm{N}$-terminal domain of Ure2p increased the glutaredoxin activity, ${ }^{64}$ suggesting that the N-terminal domain is an important feature for the proper biological function of Ure2p. ${ }^{62}$

Altogether, these data suggest that yeast $\mathrm{PrDs}$ contribute to the normal biological function of their corresponding proteins, although the extent of this contribution may not be easily detectable under normal growth conditions. Furthermore, no evidence supports a model where the evolutionary conservation of $\mathrm{N}$-terminal extensions within Sup35p and Ure $2 p$ is correlated to a necessity to maintain the prion function of these proteins. Indeed, while many (but not all) close or distant Sup35p and Ure2p orthologs have been shown to have prion forming abilities when expressed in Saccharomyces cerevisiae, evidence for such prion propagation in their natural hosts is rather scarce. ${ }^{66-73}$ This may be due to unique properties (e.g., molecular chaperones, quality-control sub-cellular compartments, etc.,) evolved by $S$. cerevisiae laboratory strains that are required for the maintenance and propagation of prions.

Why do mutations in the "non-prion" domain affect [URE3] and $\left[\mathrm{PSI}^{+}\right]$prion propagation? The effects of mutating Sup35p and Ure $2 \mathrm{p}$ PrDs on the propagation of $\left[\mathrm{PSI}^{+}\right]$and [URE3] have been extensively documented. However, few studies attempted to investigate the effects of mutating the non-PrDs of these proteins on prion propagation.

In a recent study, we showed for the first time that a single amino-acid change within the C-terminal domain of Sup35p dramatically affects its prion properties. ${ }^{7}$ The substitution of threonine 341 for alanine (Sup35A) or aspartate (Sup35D) was shown to affect the efficiency of translation termination by reducing Sup35p's GTPase activity and binding to Sup45p. ${ }^{51}$ Remarkably, these substitutions induced conformation changes within soluble Sup35p that affected its rate of assembly into fibrils. ${ }^{7}$ Furthermore, cross-seeding between Sup35p and Sup35A was inefficient, both in vitro and in vivo, suggesting that the T341A mutation alters the structural properties of Sup35p within the fibrils and generates a new prion variant. ${ }^{7}$ Indeed, we were able to show that Sup35p and Sup35A fibrils have markedly different infectious properties, and that the $\left[\mathrm{PSI}^{+}\right]$variant propagated in cells expressing only Sup35A was weaker than that of wild-type cells. ${ }^{7}$

Similarly, mutations in the "non-prion" C-terminal domain of Ure $2 p$ have been described that affect [URE3] formation. ${ }^{11}$ The deletion of residues 151-158 or a truncation of the extreme C-terminal residues (348-354) were found to increase [URE3] induction by two orders of magnitude. ${ }^{11}$ Furthermore, an ure 2 allele lacking most of the N-terminal PrD (residues 1-65) as well as residues 151-158 and 348-354 was still able to efficiently induce [URE3] when overexpressed, whereas a ure 2 allele only lacking residues 1-65 (with or without residues 348-354) could not. ${ }^{11}$ Furthermore, the deletion of residues $151-158$, that have no major consequences on Ure2p structure, accelerates the nucleation, growth and fragmentation of Ure $2 p$ fibrils in vitro, ${ }^{74}$ while the deletion of residues 221-227 resulted in a decreased ability to induce [URE3]. ${ }^{11}$ These mutational analyses suggest an interplay between the prion and "non-prion" domains of Ure2p.

Similarly, mutations have been identified in the "non-prion" domain of Rnqlp that destabilize the $\left[\mathrm{PIN}^{+}\right]$prion. ${ }^{75}$

Three events could account for the interference of the globular C-terminal domains of Sup35p and Ure2p in the assembly process. Firstly, the C-terminal domains could interact with the N-terminal domains in the soluble form of the proteins. ${ }^{7,48,60,76}$ Such an interaction should impact the ensemble 

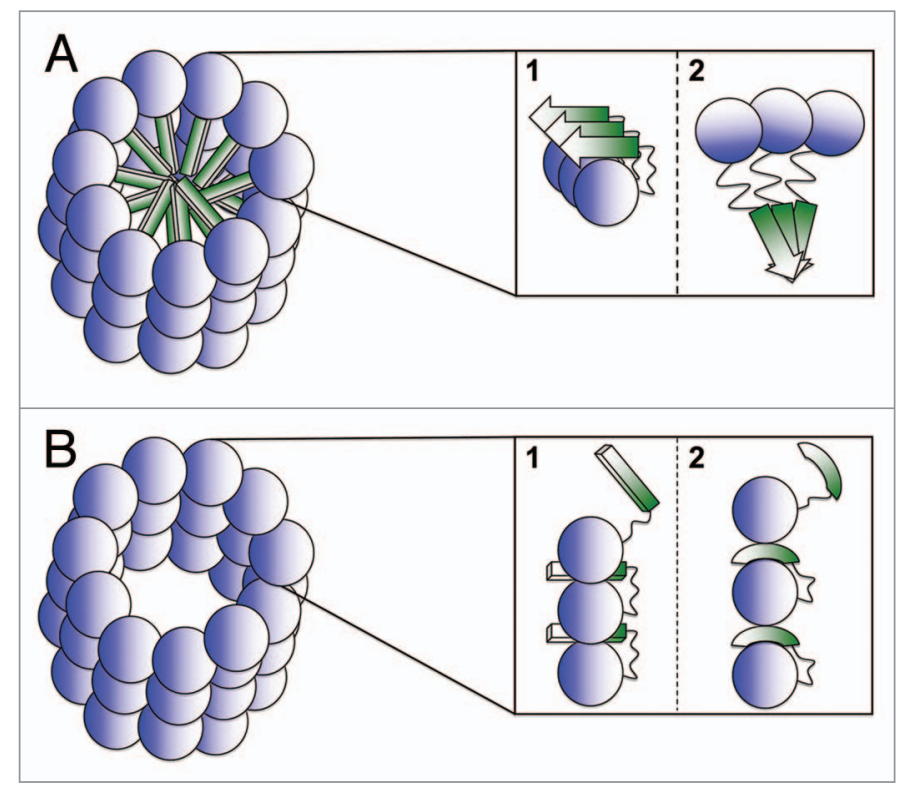

Figure 3. Schematic models of how the non-prion domains may contribute to fibrillar assemblies. (A) In this first model, the PrDs (in green) drive assembly by docking the molecules on top of each other through stacks of $\beta$-sheets. The non-PrDs (in blue) either (1) lock the structure or (2) are simply organized around a central core made by the PrD. (B) In this second model, both the PrDs and non-PrDs are integral components of the fibrillar scaffold. The PrDs may (1) interact with two adjacent non-PrDs molecules or (2) act as molecular glue between adjacent subunits.

of assembly-competent folding intermediates that can be populated by the prion domains of Sup35p or Ure2p. Secondly, conformational changes within the C-terminal domains of Sup35p and Ure2p may affect the packing of the prion domains during assembly. Thirdly, the globular C-terminal domain of Sup35p could be directly involved in the fibrillar scaffold as it is the case for Ure2p. ${ }^{29,58,60,76}$ Plausible models of how the nonPrDs of Sup $35 p$ and Ure2p may contribute to the architecture of their fibrillar assemblies are depicted in Figure 3. The fact that the PrDs of Sup35p and Ure2p have different assembly, structural and infectious properties in isolation and in the context of the full-length proteins fully supports a model where the C-terminal domain is an important part of the bona fide prion. ${ }^{29,30}$ The consequences on the structural nature of infectious prion assemblies are discussed in the following section of this review.

\section{Relationship between Prion Particles Structure and Infectivity}

To date, a limited number of direct evidence supports the tacit assumption that prions are 'infectious amyloids'. We review in the following section the molecular nature of the infectious form of prions and the relationship between the amyloid forms of prions that can be created and studied in vitro and the infectious form generated and transmitted in vivo.
What are amyloids and what is the evidence that yeast prions are amyloids? An amyloid is formally defined as a protein found in fibrillar form in extracellular deposits, which binds Congo Red and exhibits yellow-green birefringence in polarized light. ${ }^{77,78}$ This strict definition has been extended to intracellular assemblies e.g., neurofibrillary tangles that are named "amyloid-like". The amyloid fibrils have a core region consisting of $\beta$-strands that are orientated perpendicularly to the axis of the fibril that generate the characteristic diffraction pattern of X-rays by cross- $\beta$ structures. The cross- $\beta$ structure is however not diagnostic of an amyloid since amorphous protein aggregates can also show enrichment in such $\beta$-structure.79

Yeast prion domains are particularly rich in $\mathrm{Q}$ and $\mathrm{N}$ residues (Fig. 2). There is evidence that $\mathrm{Q} / \mathrm{N}$-rich regions function as "polar zippers" mediating protein-protein interactions by the capacity of the $\mathrm{Q} / \mathrm{N}$ side chains to form networks of hydrogen bonds. ${ }^{80}$ This however does not imply that these regions form cross- $\beta$ structures even though the peptide GNN QQN Y, found within the N-terminus of the prion Sup35p, was used to generate the first high resolution X-ray structure of an amyloid fibril. ${ }^{81}$ Indeed, over 100 proteins in the yeast proteome encompassing a range of protein types including transcription factors (e.g., Snf5p) and protein kinases (e.g., Yck1p) have Q/N-rich regions. ${ }^{82}$

To consider the validity of the assumption that yeast prion proteins can form amyloids, a careful analysis of both in vitro and in vivo data is required. In vitro studies have largely focused on recombinant fragments of the relevant proteins: Sup $35 \mathrm{~N}$ (1-123), Sup35NM (1-254), Ure2p (1-93) and Rnq1p (132405) (Fig. 2). In vitro, Sup35N, Sup35NM, Ure2p (1-70) alone or fused with reporter proteins and Rnq1p (132-405) fibrils are of amyloid nature., ${ }^{53,24,29,83-86}$ Full-length Sup35p and Ure2p can also assemble into fibrils (Fig. 1)..$^{24,87,88}$ These differ significantly however from those of Sup35NM and Ure2p (1-93), respectively (Fig. 1). Indeed, when the assembly of Sup35NM and full-length Sup35p are compared, significant differences in the critical concentration for assembly, the minimal size of the nuclei and the cross-seeding efficiencies are observed. ${ }^{30}$ These differences suggest either that Sup35NM or full-length Sup35p assemble into distinct fibrils, or that the C-terminal domain of Sup35p contributes to the assembly process (Fig. 3). Similarly, fibrillar Ure2p (1-93) and full-length Ure2p do not cross-seed, ${ }^{29}$ suggesting that they differ structurally.

Two forms of Ure2p fibrils have been reported: one with an $\alpha$-helical content, an X-ray fiber diffraction, solid state NMR, $\mathrm{H} / \mathrm{D}$ exchange and proteolytic patterns that are not those of a classical amyloids; $;^{58,60,87,89}$ another that has the typical characteristics of amyloids. ${ }^{85,90}$ The latter form may be the consequence of fibril dehydration. ${ }^{85}$ It is worth noting that the weak $4.7 \AA$ reflection that characterizes amyloids observed in hydrated fibrils ${ }^{90}$ may also originate from the contamination of full-length hexahistidine-tagged Ure2p preparations by the $\mathrm{N}$-terminal fragment of the protein as observed. ${ }^{86}$

Finally, although fusing Sup35p PrD to GFP certainly leads to the appearance of discrete molecular aggregates ('foci') ${ }^{35}$ in $\left[\mathrm{PSI}^{+}\right]$cells and the overexpression of Sup35p PrD generates 
"fibre-like" structures, ${ }^{91}$ the evidence that Sup35p and Ure2p can form amyloid aggregates in vivo is limited.

What makes an amyloid infectious? The amyloid state of a protein differs radically from its soluble state. Indeed, while $\beta$-sheet-containing polypeptides such as immunoglobulin can assemble into $\beta$-sheet rich amyloid fibrils, natively unfolded polypeptides such as $\alpha$-synuclein ${ }^{92}$ and even $\alpha$-helical polypeptides, such as myoglobin ${ }^{93}$ can also do so. All amyloid fibrils have a specific type of conformational arrangement of the polypeptide backbone in common, where the $\beta$-strands extend transversely to the main fibril axis while the $\beta$-sheets are parallel to the fibrils axis. ${ }^{94}$ In the cross- $\beta$ structure, the protein chains that run orthogonal to the fibril direction are hydrogen bonded, with an interchain spacing of $0.47 \mathrm{~nm}$. The peptide chains may be arranged in an antiparallel fashion, connected by reverse turns, or in a parallel fashion. Antiparallel $\beta$-sheets exhibit a crystallographic repeat that is twice the characteristic $0.47 \mathrm{~nm}$ intrasheet interchain spacing of all amyloids.

Amyloid fibrils grow indefinitely by incorporating the constituent polypeptide chains at their ends. As hydrogen bonding is the driving force of amyloid fibril growth, the two ends of the fibrils are expected to have identical or near identical growth rates. The limiting step in the assembly reaction is the conformational change that a polypeptide chain must undergo to become capable of being incorporated at one of the ends of the fibrils. While the sequence features that make an amyloid infectious remain relatively unclear, the ability of amyloid fibrils to grow indefinitely and their resistance to disassembly/degradation account at least in part for their ability to propagate.

What feature makes prion proteins infectious? Some insight into this question has come from introduction of fibrillar assemblies made in vitro into yeast cells, and the observation that the fibrils promote $\left[\mathrm{PRION}^{+}\right]$trait appearance, ${ }^{5,6,8,9}$ and a dissection of the so-called prion-forming region (Sup35N). The latter studies have demonstrated that a region containing a series of oligopeptide repeats (Fig. 2) is required for the propagation of the aggregated prion state of Sup35p, and the number of such repeats can influence the frequency of both de novo and seeded formation of the $\left[\mathrm{PSI}^{+}\right]$prion. ${ }^{95}$ Furthermore, a single amino acid substitution in one of these repeats leads to a defect in the ability to propagate the prion form of Sup35p (i.e., $\left[P S I^{+}\right]$) but not aggregation of Sup35p per se. Yet it has been suggested that the key feature of this region, and the equivalent PrD in Ure $2 p$ (residues 1-93), is the amino acid composition and not the primary amino acid sequence. This conclusion was reached by randomising the relevant prion domain sequences without changing the amino acid composition and demonstrating that the 'shuffled' PrDs could not only mediate prion propagation, but also form amyloid-like fibrils in vitro. ${ }^{9-99}$ The scrambled prions were however defective in cross seeding between the different scrambled domains in the case of Sup35p in contrast to Ure2p. ${ }^{98}$

Is prion infectivity the consequence of their potential amyloid nature or their ability to assemble? There is a widely held view that prion propagation is a result of the assembly of prions into amyloid fibrils and that the infectious and transmissible properties of these fibrils are the consequence of their capacity to act as seeds, i.e., elongate through the incorporation of soluble intracellular prion molecules in an irreversible manner. It is the generation of short fibrils, either by breakage of pre-existing fibrils or their de novo formation that is believed to drive prion propagation.

The capacity of a polypeptide to assemble cannot be used to simply describe infectivity and propagation as other protein polymers (e.g., microtubules, actin filaments, flagellin), possibly involved in the cell-to-cell transmission of epigenetic traits, ${ }^{100}$ do not define phenotypic traits. Neither can the efficiency of elongation of preexisting nuclei be at the origin of the infectious properties of prions. The only property that differentiates protein polymers that are "infectious" from those that are not, is the reversibility and strength of the protein polymers generated upon assembly. Indeed, microtubules and actin filaments are intrinsically labile polymers. Their reversibility is due to an irreversible reaction (bound nucleotide, ATP or GTP hydrolysis) occurring during assembly, the only purpose of which is the destabilization of the polymer. ${ }^{101-104}$ Prions form highly stable polymers following a conformational change, the extent of which can be variable. The establishment of numerous hydrogen bonds, hydrophobic and van der Waals interactions between polypeptides in the same conformation generates nearly irreversible polymers as the intermolecular interactions by far outweight the entropic energy in solution under physiological conditions. The extent of the conformational change and nature of intermolecular interactions are the two parameters that define the stability of such protein polymers, allowing the depolymerization of some under very mildly non-physiological conditions (e.g., flagellin, Sickle cell hemoglobin), while very harsh conditions are needed to disassemble others (e.g., Sup35p, Ure2p and Rnq1p PrDs). Thus, while the assembly propensity appears critical for prion propagation, prion assemblies do not need to be of amyloid nature to be infectious. Indeed, the establishment of a vast number of specific interactions can lead to highly stable, quasi irreversible assemblies that grow indefinitely by incorporation of the constitutive form of prion proteins.

The different domains of yeast prions, assembly and infectivity. The evidence so far suggests that fragments of the three yeast prion proteins Sup35p, Ure2p and Rnq1p can form amyloids. However, limited evidence exists for an in vivo mechanism for generating such prion fragments, nor that prion protein degradation is closely associated with the de novo formation of the associated prion traits. Furthermore, little data is available for the full-length protein molecules and where available, this data does not necessarily indicate that the resulting fibrils are amyloid in nature. It is therefore tempting to imagine that the parts of the molecules missing in most in vitro studies could influence the overall structure of the fibrils formed (Fig. 3). Evidence for the involvement of the C-terminal domains of Ure $2 p$ and Sup35p in fibril formation beside that of the N-terminal domains has been brought. ${ }^{7,59,79}$ Of particular interest is the finding that single point mutations within the compactly folded C-terminal domain of prion molecules are involved in strain formation.? 


\section{Conclusions}

Fibrillar full-length or truncated Sup35p, Ure2p and Rnq1p can induce the appearance of $\left[\mathrm{PSI}^{+}\right]$, [URE3] and $\left[\mathrm{PIN}^{+}\right]$, respectively. It is unclear whether the polymers generated in vitro physically seed the assembly of the intracellular pool of soluble prions when introduced within the cells, or induce the de novo appearance of these traits by perturbing molecular chaperones homeostasis.

Irreversible reactions designed to occur during protein assembly generate intrinsically unstable polymers that remain assembled as long as a stabilizing cap is at their ends. The loss of the caps allows the fibrils to disassemble and their constituent polypeptide to recycle. This energy consuming process allows the building of highly labile structures such as the cytoskeleton, that the cell uses to adapt to its changing environment. Although energy consuming, this system is much more economical than protein synthesis where polypeptide chains are used for a particular function only once. Highly labile, cytoskeletal proteins, although capable of polymerizing as efficiently if not better than polypeptides involved in disease, are unsuitable for the propagation of a trait. In contrast, static polymers assembled following conformational changes are made irreversible because of the establishment of a vast number of interactions which lead to specific traits. The irreversible assembly of proteins certainly contributes to make a trait heritable. Extrinsic factor(s), e.g., partner proteins, certainly play key roles in prion trait appearance and propagation as well. The interaction of both prion and "non-prion" domains of yeast prions with partner proteins in vivo may also modulate assembly. Thus, beside the need for further structural characterization of Sup35p, Ure2p and Rnq1p assemblies isolated from cell-free extracts, it is critical to characterize the contribution of prion-partner proteins by proteomic, biochemical and structural approaches to better understand the molecular events leading to prion occurrence and propagation.

\section{Acknowledgments}

Work in our laboratory is supported by the Centre National de la Recherche Scientifique (CNRS) and the Agence Nationale de la Recherche (ANR).

\section{References}

1. Tuite MF, Serio TR. The prion hypothesis: from biological anomaly to basic regulatory mechanism. Nat Rev Mol Cell Biol 2010; 11:823-33.

2. Crow ET, Li L. Newly identified prions in budding yeast, and their possible functions. Semin Cell Dev Biol 2011.

3. Halfmann R, Lindquist S. Epigenetics in the extreme: prions and the inheritance of environmentally acquired traits. Science 2010; 330:629-32.

4. Halfmann R, Alberti S, Lindquist S. Prions, protein homeostasis and phenotypic diversity. Trends Cell Biol 2010; 20:125-33.

5. Patel BK, Liebman SW. "Prion-proof" for $\left[\mathrm{PIN}^{+}\right]$: infection with in vitro-made amyloid aggregates of Rnq1p-(132-405) induces [PIN ${ }^{+}$. J Mol Biol 2007; 365:773-82.

6. Brachmann A, Baxa U, Wickner RB. Prion generation in vitro: amyloid of Ure2p is infectious. EMBO J 2005; 24:3082-92.

7. Kabani M, Cosnier B, Bousset L, Rousset JP, Melki R, Fabret C. A mutation within the C-terminal domain of Sup35p that affects $[\mathrm{PSI}(+)]$ prion propagation. Mol Microbiol 2011; 81:640-58.

8. King CY, Diaz-Avalos R. Protein-only transmission of three yeast prion strains. Nature 2004; 428:319-23.

9. Tanaka M, Chien P, Naber N, Cooke R, Weissman JS. Conformational variations in an infectious protein determine prion strain differences. Nature 2004; 428:323-8.

10. Ter-Avanesyan MD, Kushnirov VV, Dagkesamanskaya AR, Didichenko SA, Chernoff YO, Inge-Vechtomov SG, et al. Deletion analysis of the SUP35 gene of the yeast Saccharomyces cerevisiae reveals two non-overlapping functional regions in the encoded protein. Mol Microbiol 1993; 7:683-92

11. Maddelein ML, Wickner RB. Two prion-inducing regions of Ure $2 \mathrm{p}$ are nonoverlapping. Mol Cell Biol 1999; 19:4516-24.

12. Bousset L, Savistchenko J, Melki R. Assembly of the asparagine- and glutamine-rich yeast prions into protein fibrils. Curr Alzheimer Res 2008; 5:251-9.

13. Inge-Vechtomov SG, Zhouravleva GA, Chernoff YO. Biological roles of prion domains. Prion 2007; 1: 228-35.

14. Yang W, Dunlap JR, Andrews RB, Wetzel R. Aggregated polyglutamine peptides delivered to nuclei are toxic to mammalian cells. Hum Mol Genet 2002; 11:2905-17.
15. Meyer-Luehmann M, Coomaraswamy J, Bolmont T, Kaeser S, Schaefer C, Kilger E, et al. Exogenous induction of cerebral beta-amyloidogenesis is governed by agent and host. Science 2006; 313:1781-4.

16. Frost B, Jacks RL, Diamond MI. Propagation of tau misfolding from the outside to the inside of a cell. J Biol Chem 2009; 284:12845-52.

17. Li JY, Englund E, Holton JL, Soulet D, Hagell P, Lees $\mathrm{AJ}$, et al. Lewy bodies in grafted neurons in subjects with Parkinson's disease suggest host-to-graft disease propagation. Nat Med 2008; 14:501-3.

18. Ren PH, Lauckner JE, Kachirskaia I, Heuser JE, Melki R, Kopito RR. Cytoplasmic penetration and persistent infection of mammalian cells by polyglutamine aggregates. Nat Cell Biol 2009; 11:219-25.

19. Munch C, O'Brien J, Bertolotti A. Prion-like propagation of mutant superoxide dismutase-1 misfolding in neuronal cells. Proc Natl Acad Sci USA 2011; 108:3548-53.

20. Clavaguera F, Bolmont T, Crowther RA, Abramowski D, Frank S, Probst A, et al. Transmission and spreading of tauopathy in transgenic mouse brain. Nat Cell Biol 2009; 11:909-13.

21. Desplats P, Lee HJ, Bae EJ, Patrick C, Rockenstein $\mathrm{E}$, Crews $\mathrm{L}$, et al. Inclusion formation and neuronal cell death through neuron-to-neuron transmission of alpha-synuclein. Proc Natl Acad Sci USA 2009; 106:13010-5.

22. Brundin P, Melki R, Kopito R. Prion-like transmission of protein aggregates in neurodegenerative diseases. Nat Rev Mol Cell Biol 2010; 11:301-7.

23. Taylor KL, Cheng N, Williams RW, Steven AC, Wickner RB. Prion domain initiation of amyloid formation in vitro from native Ure2p. Science 1999; 283:1339-43.

24. Glover JR, Kowal AS, Schirmer EC, Patino MM, Liu JJ, Lindquist S. Self-seeded fibers formed by Sup35, the protein determinant of [PSI$\left.{ }^{+}\right]$, a heritable prion-like factor of S. cerevisiae. Cell 1997; 89:811-9.

25. Paushkin SV, Kushnirov VV, Smirnov VN, TerAvanesyan MD. In vitro propagation of the prion-like state of yeast Sup35 protein. Science 1997; 277:381-3.

26. Li L, Lindquist $\mathrm{S}$. Creating a protein-based element of inheritance. Science 2000; 287:661-4

27. Si K, Lindquist S, Kandel ER. A neuronal isoform of the aplysia CPEB has prion-like properties. Cell 2003; 115:879-91.
28. Taneja V, Maddelein ML, Talarek N, Saupe SJ, Liebman SW. A non-Q/N-rich prion domain of a foreign prion, [Het-s], can propagate as a prion in yeast. Mol Cell 2007; 27:67-77.

29. Bousset L, Bonnefoy J, Sourigues Y, Wien F, Melki R. Structure and assembly properties of the $\mathrm{N}$-terminal domain of the prion Ure $2 p$ in isolation and in its natural context. PLoS One 2010; 5:9760.

30. Krzewska J, Tanaka M, Burston SG, Melki R. Biochemical and functional analysis of the assembly of full-length Sup35p and its prion-forming domain. J Biol Chem 2007; 282:1679-86.

31. Aigle M, Lacroute F. Genetical aspects of [URE3], a non-mitochondrial, cytoplasmically inherited mutation in yeast. Mol Gen Genet 1975; 136:327-35.

32. Cox BS. PSI, a cytoplasmic suppressor of super-supressor in yeast. Heredity 1965; 20:505-21.

33. Cox BS, Tuite MF, McLaughlin CS. The psi factor of yeast: a problem in inheritance. Yeast 1988; 4:159-78.

34. Wickner RB. [URE3] as an altered URE2 protein: evidence for a prion analog in Saccharomyces cerevisiae. Science 1994; 264:566-9.

35. Patino MM, Liu JJ, Glover JR, Lindquist S. Suppor for the prion hypothesis for inheritance of a phenotypic trait in yeast. Science 1996; 273:622-6.

36. Derkatch IL, Bradley ME, Hong JY, Liebman SW. Prions affect the appearance of other prions: the story of [PIN(+)]. Cell 2001; 106:171-82.

37. Derkatch IL, Bradley ME, Masse SV, Zadorsky SP, Polozkov GV, Inge-Vechtomov SG, et al. Dependence and independence of $[\mathrm{PSI}(+)]$ and $[\mathrm{PIN}(+)]$ : a twoprion system in yeast? EMBO J 2000; 19:1942-52.

38. Derkatch IL, Bradley ME, Zhou P, Chernoff YO, Liebman SW. Genetic and environmental factors affecting the de novo appearance of the $\left[\mathrm{PSI}^{+}\right]$prion in Saccharomyces cerevisiae. Genetics 1997; 147:507-19.

39. Coschigano PW, Magasanik B. The URE2 gene product of Saccharomyces cerevisiae plays an important role in the cellular response to the nitrogen source and has homology to glutathione s-transferases. Mol Cell Biol 1991; 11:822-32

40. Cox KH, Rai R, Distler M, Daugherty JR, Coffman JA, Cooper TG. Saccharomyces cerevisiae GATA sequences function as TATA elements during nitrogen catabolite repression and when Gln3p is excluded from the nucleus by overproduction of Ure2p. J Biol Chem 2000; 275:17611-8. 
41. Kulkarni AA, Abul-Hamd AT, Rai R, El Berry H, Cooper TG. Gln3p nuclear localization and interaction with Ure2p in Saccharomyces cerevisiae. J Biol Chem 2001; 276:32136-44.

42. Chernoff YO, Lindquist SL, Ono B, Inge-Vechtomov SG, Liebman SW. Role of the chaperone protein Hsp104 in propagation of the yeast prion-like factor [psi ${ }^{+}$. Science 1995; 268:880-4.

43. Moriyama H, Edskes HK, Wickner RB. [URE3] prion propagation in Saccharomyces cerevisiae: requirement for chaperone Hsp104 and curing by overexpressed chaperone Ydj1p. Mol Cell Biol 2000; 20:8916-22.

44. Derkatch IL, Chernoff YO, Kushnirov VV, IngeVechtomov SG, Liebman SW. Genesis and variability of [PSI] prion factors in Saccharomyces cerevisiae. Genetics 1996; 144:1375-86.

45. Inagaki Y, Ford Doolittle W. Evolution of the eukaryotic translation termination system: origins of release factors. Mol Biol Evol 2000; 17:882-9.

46. Resende CG, Outeiro TF, Sands L, Lindquist S, Tuite MF. Prion protein gene polymorphisms in Saccharomyces cerevisiae. Mol Microbiol 2003; 49: 1005-17.

47. Harrison LB, Yu Z, Stajich JE, Dietrich FS, Harrison PM. Evolution of budding yeast prion-determinant sequences across diverse fungi. J Mol Biol 2007; 368:273-82.

48. Kong C, Ito K, Walsh MA, Wada M, Liu Y, Kumar S, et al. Crystal structure and functional analysis of the eukaryotic class II release factor eRF3 from $S$. pombe. Mol Cell 2004; 14:233-45.

49. Le Goff C, Zemlyanko O, Moskalenko S, Berkova N, Inge-Vechtomov S, Philippe M, et al. Mouse GSPT2, but not GSPT1, can substitute for yeast eRF3 in vivo. Genes Cells 2002; 7:1043-57.

50. Gagny B, Silar P. Identification of the genes encoding the cytosolic translation release factors from Podospora anserina and analysis of their role during the life cycle. Genetics 1998; 149:1763-75.

51. Fabret C, Cosnier B, Lekomtsev S, Gillet S, Hatin I, Le Marechal P, et al. A novel mutant of the Sup35 protein of Saccharomyces cerevisiae defective in translation termination and in GTPase activity still supports cell viability. BMC Mol Biol 2008; 9:22.

52. Volkov K, Osipov K, Valouev I, Inge-Vechtomov S, Mironova L. N-terminal extension of Saccharomyces cerevisiae translation termination factor eRF3 influences the suppression efficiency of sup35 mutations. FEMS Yeast Res 2007; 7:357-65.

53. Urakov VN, Valouev IA, Kochneva-Pervukhova NV, Packeiser AN, Vishnevsky AY, Glebov OO, et al. $\mathrm{N}$-terminal region of Saccharomyces cerevisiae eRF3 is essential for the functioning of the eRF1/eRF3 complex beyond translation termination. BMC Mol Biol 2006; 7:34.

54. Hosoda N, Kobayashi T, Uchida N, Funakoshi Y, Kikuchi Y, Hoshino S, et al. Translation termination factor eRF3 mediates mRNA decay through the regulation of deadenylation. J Biol Chem 2003; 278: 38287-91.

55. Umland TC, Taylor KL, Rhee S, Wickner RB, Davies DR. The crystal structure of the nitrogen regulation fragment of the yeast prion protein Ure2p. Proc Natl Acad Sci USA 2001; 98:1459-64.

56. Bousset L, Belrhali H, Janin J, Melki R, Morera S. Structure of the globular region of the prion protein Ure2 from the yeast Saccharomyces cerevisiae. Structure 2001; 9:39-46.

57. Bousset L, Belrhali H, Melki R, Morera S. Crystal structures of the yeast prion Ure $2 p$ functional region in complex with glutathione and related compounds. Biochemistry 2001; 40:13564-73.

58. Bousset L, Thomson NH, Radford SE, Melki R. The yeast prion Ure $2 p$ retains its native alpha-helical conformation upon assembly into protein fibrils in vitro. EMBO J 2002; 21:2903-11.
59. Fay N, Redeker V, Savistchenko J, Dubois S, Bousset L, Melki R. Structure of the prion Ure2p in protein fibrils assembled in vitro. J Biol Chem 2005; 280:37149-58.

60. Loquet A, Bousset L, Gardiennet C, Sourigues Y, Wasmer C, Habenstein B, et al. Prion fibrils of Ure2p assembled under physiological conditions contain highly ordered, natively folded modules. J Mol Biol 2009; 394:108-18.

61. Masison DC, Wickner RB. Prion-inducing domain of yeast Ure $2 p$ and protease resistance of Ure $2 p$ in prioncontaining cells. Science 1995; 270:93-5.

62. Shewmaker F, Mull L, Nakayashiki T, Masison DC, Wickner RB. Ure2p function is enhanced by its prion domain in Saccharomyces cerevisiae. Genetics 2007; 176:1557-65.

63. Bai M, Zhou JM, Perrett S. The yeast prion protein Ure2 shows glutathione peroxidase activity in both native and fibrillar forms. J Biol Chem 2004; 279:50025-30.

64. Zhang ZR, Perrett S. Novel glutaredoxin activity of the yeast prion protein Ure2 reveals a native-like dimer within fibrils. J Biol Chem 2009; 284:14058-67.

65. Rai R, Tate JJ, Cooper TG. Ure2, a prion precursor with homology to glutathione S-transferase, protects Saccharomyces cerevisiae cells from heavy metal ion and oxidant toxicity. J Biol Chem 2003; 278:12826-33.

66. Kushnirov VV, Kochneva-Pervukhova NV, Chechenova MB, Frolova NS, Ter-Avanesyan MD. Prion properties of the Sup35 protein of yeast Pichia methanolica. EMBO J 2000; 19:324-31.

67. Nakayashiki T, Ebihara K, Bannai H, Nakamura Y. Yeast $\left[\mathrm{PSI}^{+}\right]$"prions" that are crosstransmissible and susceptible beyond a species barrier through a quasiprion state. Mol Cell 2001; 7:1121-30.

68. Santoso A, Chien P, Osherovich LZ, Weissman JS Molecular basis of a yeast prion species barrier. Cell 2000; 100:277-88.

69. Tanaka M, Chien P, Yonekura K, Weissman JS. Mechanism of cross-species prion transmission: an infectious conformation compatible with two highly divergent yeast prion proteins. Cell 2005; 121:49-62.

70. Chernoff YO, Galkin AP, Lewitin E, Chernova TA, Newnam GP, Belenkiy SM. Evolutionary conservation of prion-forming abilities of the yeast Sup35 protein. Mol Microbiol 2000; 35:865-76.

71. Edskes HK, Engel A, McCann LM, Brachmann A Tsai HF, Wickner RB. Prion-forming ability of Ure2 of yeasts is not evolutionarily conserved. Genetics 2011; 188:81-90.

72. Talarek N, Maillet L, Cullin C, Aigle M. The [URE3] prion is not conserved among Saccharomyces species. Genetics 2005; 171:23-34.

73. Safadi RA, Talarek N, Jacques N, Aigle M. Yeast prions: could they be exaptations? The URE2/[URE3] system in Kluyveromyces lactis. FEMS Yeast Res 2011; 11: 151-3.

74. Chen L, Chen LJ, Wang HY, Wang YQ, Perrett S. Deletion of a Ure2 C-terminal prion-inhibiting region promotes the rate of fibril seed formation and alters interaction with Hsp40. Protein Eng Des Sel 2011; 24:69-78.

75. Shibata S, Kurahashi H, Nakamura Y. Localization of prion-destabilizing mutations in the $\mathrm{N}$-terminal non-prion domain of Rnq1 in Saccharomyces cerevisiae. Prion 2009; 3:250-8.

76. Redeker V, Halgand F, Le Caer JP, Bousset L, Laprevote O, Melki R. Hydrogen/deuterium exchange mass spectrometric analysis of conformational changes accompanying the assembly of the yeast prion Ure2p into protein fibrils. J Mol Biol 2007; 369:1113-25.

77. Westermark P, Benson MD, Buxbaum JN, Cohen AS, Frangione B, Ikeda S, et al. Amyloid fibril protein nomenclature-2002. Amyloid 2002; 9:197-200.

78. Westermark P, Benson MD, Buxbaum JN, Cohen AS, Frangione B, Ikeda S, et al. Amyloid: toward terminology clarification. Report from the Nomenclature Committee of the International Society of Amyloidosis. Amyloid 2005; 12:1-4.
79. Bousset L, Redeker V, Decottignies P, Dubois S, Le Marechal P, Melki R. Structural characterization of the fibrillar form of the yeast Saccharomyces cerevisiae prion Ure2p. Biochemistry 2004; 43:5022-32.

80. Perutz MF, Johnson T, Suzuki M, Finch JT. Glutamine repeats as polar zippers: their possible role in inherited neurodegenerative diseases. Proc Natl Acad Sci USA 1994; 91:5355-8

81. Nelson R, Sawaya MR, Balbirnie M, Madsen AO, Riekel C, Grothe R, et al. Structure of the cross-beta spine of amyloid-like fibrils. Nature 2005; 435:773-8.

82. Michelitsch MD, Weissman JS. A census of glutamine/ asparagine-rich regions: implications for their conserved function and the prediction of novel prions. Proc Natl Acad Sci USA 2000; 97:11910-5.

83. Schlumpberger M, Wille H, Baldwin MA, Butler DA, Herskowitz I, Prusiner SB. The prion domain of yeast Ure2p induces autocatalytic formation of amyloid fibers by a recombinant fusion protein. Protein $\mathrm{Sci}$ 2000; 9:440-51.

84. Serio TR, Cashikar AG, Kowal AS, Sawicki GJ, Moslehi JJ, Serpell L, et al. Nucleated conformational conversion and the replication of conformational information by a prion determinant. Science 2000; 289:1317-21.

85. Baxa U, Cheng N, Winkler DC, Chiu TK, Davies DR, Sharma D, et al. Filaments of the Ure2p prion protein have a cross-beta core structure. J Struct Biol 2005 ; 150:170-9.

86. Baxa U, Taylor KL, Wall JS, Simon MN, Cheng $\mathrm{N}$, Wickner RB, et al. Architecture of Ure2p prion filaments: the $\mathrm{N}$-terminal domains form a central core fiber. J Biol Chem 2003; 278:43717-27.

87. Thual C, Komar AA, Bousset L, Fernandez-Bellot E, Cullin C, Melki R. Structural characterization of Saccharomyces cerevisiae prion-like protein Ure2. J Biol Chem 1999; 274:13666-74.

88. Krzewska J, Melki R. Molecular chaperones and the assembly of the prion Sup35p, an in vitro study. EMBO J 2006; 25:822-33.

89. Bousset L, Briki F, Doucet J, Melki R. The native-like conformation of Ure $2 p$ in fibrils assembled under physiologically relevant conditions switches to an amyloidlike conformation upon heat-treatment of the fibrils. J Struct Biol 2003; 141:132-42.

90. Wang YQ, Bongiovanni M, Gras SL, Perrett S. The fibrils of Ure2p homologs from Saccharomyces cerevisiae and Saccharoymyces paradoxus have similar cross-beta structure in both dried and hydrated forms. J Struct Biol 2011; 174:505-11.

91. Kawai-Noma S, Pack CG, Kojidani T, Asakawa H, Hiraoka $Y$, Kinjo $M$, et al. In vivo evidence for the fibrillar structures of Sup35 prions in yeast cells. J Cell Biol 2010; 190:223-31.

92. Uversky VN, Li J, Fink AL. Evidence for a partially folded intermediate in alpha-synuclein fibril formation. J Biol Chem 2001; 276:10737-44.

93. Fandrich M, Fletcher MA, Dobson CM. Amyloid fibrils from muscle myoglobin. Nature 2001; 410 165-6.

94. Blake C, Serpell L. Synchrotron X-ray studies suggest that the core of the transthyretin amyloid fibril is a continuous beta-sheet helix. Structure 1996; 4:989-98.

95. Tuite MF, Cox BS. The genetic control of the formation and propagation of the $\left[\mathrm{PSI}^{+}\right]$prion of yeast. Prion 2007; 1:101-9.

96. Ross ED, Baxa U, Wickner RB. Scrambled prion domains form prions and amyloid. Mol Cell Biol 2004; 24:7206-13.

97. Ross ED, Edskes HK, Terry MJ, Wickner RB. Primary sequence independence for prion formation. Proc Natl Acad Sci USA 2005; 102:12825-30.

98. Ross CD, McCarty BR, Hamilton M, Ben-Hur A, Ross ED. A promiscuous prion: efficient induction of [URE3] prion formation by heterologous prion domains. Genetics 2009; 183:929-40. 
99. Toombs JA, Liss NM, Cobble KR, Ben-Musa Z, Ross ED. [PSI] Maintenance Is Dependent on the Composition, Not Primary Sequence, of the Oligopeptide Repeat Domain. PLoS One 2011; 6:21953.

100. Ganusova EE, Ozolins LN, Bhagat S, Newnam GP, Wegrzyn RD, Sherman MY, et al. Modulation of prion formation, aggregation and toxicity by the actin cytoskeleton in yeast. Mol Cell Biol 2006; 26:617-29.
101. Mitchison T, Kirschner M. Dynamic instability of microtubule growth. Nature 1984; 312:237-42.

102. Carlier MF, Melki R, Pantaloni D, Hill TL, Chen Y. Synchronous oscillations in microtubule polymerization. Proc Natl Acad Sci USA 1987; 84:5257-61.
103. Wegner A. Head to tail polymerization of actin. J Mol Biol 1976; 108:139-50.

104. Korn ED, Carlier MF, Pantaloni D. Actin polymerization and ATP hydrolysis. Science 1987; 238:638-44. 